\title{
Evaluation the effect of progressive muscle relaxation technique on fatigue and daily living activities in patients with Multiple Sclerosis
}

\author{
TAYEBEH JAVDAN ${ }^{1}$, ELHAM IMANI ${ }^{*}$, AHMAD AGHA NEGAHI ${ }^{3}$, SAEED HOSSEINI TESHNIZI ${ }^{4}$ \\ ${ }^{1}$ Master of Nursing, Student Research Committee, Hormozgan University of Medical Sciences, Bandar Abbas, Iran, ORCID ID: 0000-0002- \\ 9173-1014 \\ ${ }^{2}$ Assistant Professor, Nursing Department, Hormozgan University of Medical Sciences, Bandar Abbas, Iran, ORCID ID: 0000-0003-3957- \\ 3343 \\ ${ }^{3}$ Assistant Professor, Neurology and Internal Medicine Department, Hormozgan University of Medical Sciences, Bandar Abbas, Iran, ORCID \\ ID: 0000-0003-4369-2468 \\ ${ }^{4}$ Assistant Professor, Biostatistics Department, Hormozgan University of Medical Sciences, Bandar Abbas, Iran, ORCID ID: 0000-0002- \\ 5575-6855 \\ ${ }^{\star}$ Corresponding author: Elham Imani, Phone number: 00989179397802, FAX number 00987632581180, Email: Eimani@hums.ac.ir
}

\begin{abstract}
Aim: This is a Quasi-experimental study wich was carried out in order to determine the effects of applying progressive muscle relaxation on fatigue and daily living activity of MS patients.

Background: Fatigue is one of the most common symptoms in people with multiple sclerosis (MS) and adversely affects their daily living activities.

Methods: 80 MS Patients were selected then conducted in two group (40 experiments and 40 contorol). Data collector tools included: demographic questionnaire, fatigue severity scale and activity of daily living questionnaire which completed four times by two group (before Third week, sixth and ninth after study), self-report checklists which completed only by experimental group. PMRT performed once a day for 8 week by experimental group. During study no intervention was done for control group.

Results: repeated measures ANOVA showed that there is significant difference in mean score of fatigue between two group in 4 times $(\mathrm{P}<0 / 05)$. Also, the results showed that with progressive muscle relaxation at 4 times, daily activities in ADL and IADL sections were significantly increased $(P<0 / 05)$ but there was no significant difference between the two groups ( $P>0 / 05)$. however, clinically significant differences were observed.

Conclusions: Acording to these findings applying PMRT decreased patient's fatigue and improve independence in daily living activities. This study supports the effect of PMRT on fatigue and activities of daily living in patients with MS, and it is recommended that further studies be conducted on this subject in the future.

Keywords: daily living activity, fatigue, progressive muscle relaxation technique, multiple sclerosis.
\end{abstract}

\section{INTRODUCTION}

Multiple sclerosis is a progressive autoimmune disorder in central nervous system which affects brain and spine.

By eliminating myelin sheath of the neurons, connective signaling among brain and peripheral organs is lost, which is occurred mostly in young adults, and causes various disabilities.

This disease is not competely improved and the aim of treatment is delaying progression of the disease, treatment of chronic symptoms and acute recurrences (Kaminska, Koper, Piechal, \& Kemona, 2017).

Prevalence of MS in the world is 110 in each 100000 individuals (Charvet, Shaw, Sherman, Haas, \& Krupp, 2018).

According to the reports by Iran MS association, more than 70,000 individuals in Iran are affected by the diseases (Hooshmand, 2018).

The disease is accompanied by dysfunction in physical activities, chronic pain, fatigue, depression, and cognitive disorders.

Various studies showed association among these symptoms and various outcomes including occupational status, disability, disruption in social function, lifesatisfaction, daily activities, and physical-mental health (Kratz et al., 2017).

Given various studies, fatigue is reported during diseases period of $75-95 \%$ of the patients, and two third of these individuals know fatigue as one of the worst signs of the diseases which vigorously affects daily function and performance and life quality.

Pathologic fatigue is one of the most common leading causes of unemployment in patients with MS.

Additionally, fatigue causes limitations in social relations and self-care activities, and usually limits the person's ability to do tasks which needs physical power (Dayapoglu \& Tan, 2012).

Research showed that high prevalence of fatigue and weakness affect doing daily activities.

Disruption in living daily activities is an important and funamental issue in patients with MS, which in $69.4 \%$ of affected individuals causes limitation in doing daily activities (Ghaffari, Ahmadi, Nabavi, Me'marian, \& KazemNejad, 2007).

Fatigue and inability to do life daily activities and dependency of patients face health care team to problem.

Patients suffer severe fatigue do not participate in therapeutic decisions due to lack of sufficient energy, enthusiasm, and concentration, do not adhere thoroughly to health instructions and treatment protocols and do not communicate properly with health care team.

Due to growing increase of patients affected by MS in Iran, and due to importance of fatigue and their dependency in doing life usual activities in the patients, and since pharmaceutical treatments endure heavy load to society, family, and the patients, and due to side effects of these medications and incomplete resolving of fatigue with 
Tayebeh Javdan, Elham Imani, Ahmad Agha Negahi et al

consuming fatigue-reducing medication, as well as acceptance of complementary treatments in health and treatment systems and various diseases, using nonpharmaceutical treatments in patients with MS is recommended.

These treatments are known as alternative or complementary medicine which might be used instead of or along with pharmaceutical treatments.

Some complementary treatments in MS include: Acupuncture, feedback therapy, hypnotism, massage therapy, relaxation, Thi Chi technique, meditation and yoga.

Progressive muscle relaxation (PMR) is one of these non-pharmaceutical treatment methods.

This method is one of the complementary treatment methods presented at 1938 by Jacobson.

In this technique, the individual causes calmness in various muscles by contraction and relaxing specific muscles.

PMR regulates nervous function and increases desirable and optimum feelings and mental concentrations.

This technique is one of the nursing interventions which performing and educating this method is simple.

Saving costs, lack of need to specific facilities and simplicity of performance by the patients are among the properties of this method (Zargarani, Maghsoudlou, \& Zaman Kamkar, 2018).

Various studies are performed to investigate PMR in various diseases, for instance, the study by Saki et al at 2011 observed positive effect of this technique on anxiety in patients with heart attack.

Ghafari et al observed that performing PMR in patients with MS can reduce depression and anxiety (Jariani, Saki, Momeni, Ebrahimzade, \& Seydian, 2011).

Another study at 2017 showed that performing this technique in patients with chronic obstructive pulmonary diseases reduces fatigue and improves sleep quality (Yilmaz \& Kapucu, 2017).

Findings by Zargarzade et al at 2014 also indicates the considerable effect of PMR in reducing chronic back pain and increasing daily activity of the patients with intervertebral disck (Zargarzadeh, Memarian, \& Rafiee, 2015).

Given above issues and fundamental role of health care givers especially nurses in planning appropriate managements to improve health status and promote daily activities in patients, this study is designed based on requirements of patients referred to MS clinic of Bandar Abbas due to negligible financial load and simplicity of application, to determine effect of PMR on fatigue severity and daily activities of patients with MS.

\section{METHODS}

The current study is a quasi-experimental research conducted at the time gap between May and September 2019 at MS clinic of Bandar Abbas, the population of this study is all the patients aged more than 18 years with MS referred to MS clinic of Bandar Abbas.

Inclusion criteria were patients with MS, to have Expanded Disability Status Scale (EDSS) score $\leq 5.0$ (EDSS was evaluated by a neurologist), age more than 18 years, tendency and willingness to participate in the study, ability to read and write, lack of other chronic diseases such as Rheumatologic, neurologic, orthopedic, and ...diseases (Dayapoglu \& Tan, 2012) not being at the acute phase of the disease, being habitant of Bandar Abbas (Ghaffari et al., 2007), not being addicted to any drugs, lack of history of PMR, lack of any acute psychiatric disorders (Zargarani et al., 2018).

Exclusion criteria include lack of participation at educational sessions, refusing continuing cooperation due to any reason (Yilmaz \& Kapucu, 2017).

Sample size was determined at 38 individuals due to sample equation and by assumption of maximum first type error of $5 \%$, which due to attrition rate, the sample size was considered as 40 individuals, which 4 individuals were excluded and finally the study was conducted on 76 patients.

Selecting patients was done through convenience sampling and then were allocated to two intervention and control groups based on randomization Table.

Samples were classified into four 10-individuals group and four sessions were considered for educating PMR.

In summary, the client in order to perform PMR, sits conveniently on a chair or lies down, it is better to wear a loose cloth and remove the bracelet, watch, and ....

Then the client contracts and relaxes the various muscle groups along with deep and effective breathing, the client should continue this training until the time that he feels the difference between contraction and relaxation of muscles and express it, thereby, he learns the technique and is able to do the technique alone and using the voice file.

Then, this technique was done one daily by the participants in intervention group at home for 8 weeks.

It should be noted that no intervention was done for control group.

Gathering data tool in the current study include personal characteristics checklist, fatigue index questionnaire, daily life activities questionnaire, and selfreport checklist.

Personal characteristics checklist includes two parts. Demographic information including: age, gender, marital status, occupation, income, educational level, and information related to disease including: diseases duration, frequency of disease recurrence, and frequency of hospitalization during last year.

Fatigue severity scale (FSS) is a valid scale which assesses fatigue in general in patients with MS.

This scale consists of 9 questions which 5 questions assess quality of fatigue, 3 questions assess physical, mental fatigue and results of fatigue on individual's social status.

One question compares severity of fatigue with other symptoms in the patient with MS.

Score of each question is from 1-7. Score of 1 means that the person is greatly disagreed with the situation and score of 7 means that the individual is absolutely agreed with it.

Score of 7 in this tool showed highest fatigue and score of 1 means lack of fatigue.

In order to achieve total score of the scale, entire scores were summed, this score ranged from 9 to 63 . The higher the score, the more severe the fatigue. 
Reliability and validity of the Persian version of the FSS in individuals with MS were assessed in the study by Farahani et al, which ICC coefficient was achieved at 0.93 (A'zimian, Fallah-Pour, \& Karimlou, 2013).

Daily life activity in samples was assessed using daily life questionnaire and life tool.

This questionnaire consists of two sections related to ADL (activity of daily living) with 7 questions and IADL (instrumental activities of daily living)

Daily life activity consists: eating, wearing and taking off clothes, walking, doing works related to appearance, taking shower or bathing, going to bed, coming and going to bathroom.

Daily life activities including using telephone, going long distances with vehicles, buying food and clothes, preparing foods, doing home tasks, washing clothes, doing fixings at home, consuming medications, and managing income and expenses.

This tool measures independency in performing daily activity in a three level Likert spectrum without help=2, with a little help=1, I cannot do it $=0$.

Score ranges of activities daily life is between $0-14$, and range of scores of IADL is among $0-18$.

The higher the scores, indicates higher independency of the individual in performing daily activities and life tool and vice versa.

Validity of this tool was assessed in the study by Habibi Sola, Nikpoor, Rezaei, and Haghani (2013) through content and spontaneous validity method.

Reliability of the tool was reported using retest method $(r=0.9)$ (Habibi Sola, Nikpoor, Rezaei, \& Haghani, 2013).

Self-report checklist is a research-made checklist which is provided to clients to be assured on performing relaxation technique, to record day, time and duration of technique and in case of not doing the technique.

FSS and daily activities level in four stages of baseline, at the end of third, sixth, and ninth week after intervention in both groups of intervention and control.

Finally, the data achieved from the questionnaires was analyzed using SPSS version 22 and independent ttest, Chi-square test, repeated measures ANOVA and
Pearson correlation. Significance level was set at $\mathrm{P}<0.05$ for all tests.

\section{RESULTS}

Results related to demographic information of participants in the study showed that mean age of the participants was $36 \pm 7.94$, the highest percentage regarding gender was woman $(61.8 \%)$ and the highest percentage regarding marital status was $(60.5 \%)$.

The highest percentage regarding occupational status is housewife as $31.6 \%$, and the highest percentage had middle school and high-school degree (55.3\%).

Statistical analysis on related information showed that the average duration of the diseases is $8.20 \pm 5.15$.

Most patients $(51.3 \%)$ had not disease recurrence in last year, most participants in the intervention (73.7\%) and control $(81.6 \%)$ group had not hospitalization history in hospital in last year.

Regarding type of medication, most patients in intervention group (44.7\%) and control group (39.5\%) used combination medications (MS-modulating medications along with symptom-therapy medications).

Chi-square test for all qualitative variables and independent t-test with $P>0.05$ for all quantitative variables did not show significant difference among two intervention and control groups.

Due to random allocation of samples into two groups, it can be said that results obtained from the intervention is due to the effect of these variables on the individuals.

Independent t-test showed that there is no significant difference among mean score of fatigue and activities of daily life $(P>0.05)$.

Results of repeated measures ANOVA showed that fatigue in four stages of measurement (without considering study groups) had significant difference $(P<0.001)$.

Interaction of time and group showed a significant difference in fatigue decline in two groups $(P<0.001)$, so that fatigue at intervention time to the end of study in the intervention group is significantly lower than control group $(P<0.001)$ (Table 1).

Table 1. Results of repeated measure ANOVA to compare the mean fatigue at four measurement times in intervention and control groups.

\begin{tabular}{|l|l|l|l|l|l|l|}
\hline Variable & Source & Sum of squares & Df. & Mean square & S & \\
\hline Fatique & Time & 5141.56 & 3 & 2609.06 & 183.34 & $<0.001$ \\
& & & & & & \\
\cline { 2 - 8 } & Time*group & 4723.45 & 3 & 2396.89 & 168.43 & $<0.001$ \\
\cline { 2 - 8 } & Group & 8243.05 & 1 & 8243.05 & 34.22 & $<0.001$ \\
\hline
\end{tabular}

Results of repeated measures ANOVA showed independency of the patients in doing activities of daily life in two parts of ADL and IADL is significantly increased by doing intervention of PMR, however, significant difference is not observed among two intervention and control group $(P>0.05)$ (Tables 2, 3).

Table 2. Results of repeated measure ANOVA to compare the mean ADL at four measurement times in intervention and control groups.

\begin{tabular}{|l|l|l|l|l|l|l|}
\hline Variable & Source & Sum of squares & Df. & Mean square & $\mathbf{f}$ & Sig. \\
\hline \multirow{4}{*}{ ADL } & Time & 74.08 & 3 & 35.26 & 23.92 & $<0.001$ \\
\cline { 2 - 8 } & Time*group & 47.53 & 3 & 22.62 & 15.35 & $<0.001$ \\
\cline { 2 - 8 } & Group & 13.89 & 1 & 13.89 & 0.87 & 0.35 \\
\hline
\end{tabular}


Tayebeh Javdan, Elham Imani, Ahmad Agha Negahi et al

Table 3. Results of repeated measure ANOVA to compare the mean IADL at four measurement times in intervention and control groups.

\begin{tabular}{|c|c|c|c|c|c|c|}
\hline Variable & Source & Sum of squares & Df. & Mean square & $f$ & Sig. \\
\hline \multirow[t]{3}{*}{ IADL } & Time & 460.77 & 3 & 282.15 & 63.16 & $<0.001$ \\
\hline & Time* group & 292.19 & 3 & 178.92 & 40.05 & $<0.001$ \\
\hline & Group & 5.53 & 1 & 5.53 & 0.10 & 0.74 \\
\hline
\end{tabular}

\section{DISCUSSION}

In this study, PMR reduces fatigue in intervention group comparing to control group. This result can show the reducing effect of PMR on fatigue in patients.

The study by Rigi et al conducted to investigate reflexology on fatigue in women with MS at 2016, the results of study showed that complementary treatments such as:

The results of the study showed complementary treatments including reflex massage therapy are effective on fatigue in women with MS (Rigi, shahnavazi, Feizi, Adineh, \& Malekraisi noushirvan, 2017).

Study by Bimoradi et al at 2013 showed relaxation is effective in reducing fatigue in patients with MS (Bikmoradi, Zafari, Oshvandi, Mazdeh, \& Roshanaei, 2014).

This study in line with the current study showed the positive effect of PMR on complications of MS.

Study by Michel cava et al conducted to investigate effect of rehabilitating treatments on fatigue in patients with MS; showed that complementary methods such as rehabilitating plans is effective in reducing fatigue in patients with MS (Mikulakova, Klimova, Kendrova, Gajdos, \& Chmelik, 2018).

The study by Jalal manesh et al at 2016 showed that application of PMR in patients with MS is considerably effective in reducing fatigue (Jalal manesh \& Zargarani, 2015).

The results of the study consistent with the current study showed positive effects of PMR on fatigue severity in patients with MS.

This study was conducted on one group, however the current study was conducted on two case and control groups.

In addition, the results of the study showed that by performing PMR over time, independency of the patients in doing activities of daily life in two parts of ADL and IADL are significantly increased however no significant difference was among two case and control groups.

The lack of difference among groups might be due to chronic and progressive nature of the disease, and the relation between other symptoms of the disease such as depression and physical problems on daily activities.

In the study by Barak et al, performing exercise training along with muscle contraction and relaxation improves physical performance in patients with MS (Barak, Hutzler, Dubnov-Raz, \& Achiron, 2016).

In the study by Edward entitled "Effect of exercise training on disability of patients with MS", the exercise training promotes physical aspects of daily life activities (Edwards \& Pilutti, 2017).

The study by Sebastive et al which was conducted to assess effect of aerobic on patients with MS, showed that exercise therapy is effective on promoting daily activities in patients with MS (Sebastião, Pilutti, \& Motl, 2019).
The results obtained from the above study are inconsistent with the current study indicating lack of effect of PMR on promoting activities of daily life.

This inconsistency might be due to application of different technique, difference in intervention duration and effect of other disease symptoms on activities of daily life.

Therefore, by increasing duration of performing of PMR, the positive results might be observed.

Assessment of association among demographic variables and fatigue rate identified that, there is no significant association among age, gender, marital status, educational level, occupational status, monthly income, disease duration, frequency of hospitalization, history of underlying disease and type of medication used.

There is a significant association among fatigue and frequency of recurrence of disease in last year in the intervention group; so that in patients with more than twice recurrence, fatigue is higher comparing to patients who has less than twice recurrence.

There is positive significant association among ADL and age $(r=0.47, P=0.003)$, and disease duration $(r=0.45$, $P=0.004)$, and also among IADL and age $(r=0.43$, $P=0.007)$, and disease duration $(r=0.39, \quad P=0.01)$ in intervention group;

It means that those participants who did PMR, both levels of ADL and IADL is increased by increase in age, and dependency of individuals in performing daily activity life is decreased.

In addition, ADL and IDAL levels in individuals with longer disease duration who performed PMR was more increased comparing to those with shorter disease duration. There is significant association among ADL and IADL; so that disabled patients who had minimum level of daily life activities comparing to other occupational groups prior to intervention, showed highest increase in ADL and IADL level through doing PMR and increase in independency in doing daily activities.

In addition, there was significant association among frequency of recurrence and hospitalization in last year with two levels of daily life activities in intervention group, indicating that those individuals with one or two recurrence, showed more increase in ADL and IADL level after doing PMR comparing to those with no recurrence or more than twice of recurrence per year.

Regarding hospitalization frequency in last year, participants in intervention group who had once or twice hospitalization showed more considerable increase in ADL and IADL comparing to those with no history.

No significant association was observed among level of daily life activity and gender, marital status, educational level, monthly income, history of underlying disease and type of medication used in both groups of intervention and control in two parts of ADL and IADL. 


\section{CONCLUSIONS}

Findings of the current study showed that PMR might reduce fatigue severity efficiently in patients with MS, hence it is expected that the results of the current study might be useful for nurses working in wards related to hospitalization of patients with MS, and by applying PMR by nurses as non-pharmaceutical intervention, an effective change in order to improve nursery services and also patients' status.

Increasing duration of PMR and assessment of its effect on other symptoms of patients with MS and also other chronic diseases is recommended.

Limitations of this study include personal differences and mental status of study population in response to intervention and its effect, effect of learning and lack of mental conflict on effect rate of PMR as well as disabling nature of disease.

Funding: This work was supported by the Hormozgan University of Medical Sciences [grant numbers 970287].

Conflicts of interest: The authors declare that they have no conflict of interest.

\section{REFERENCES}

1. A'zimian, M., Fallah-Pour, M., \& Karimlou, M. (2013). Evaluation of reliability and validity of the Persian version of Fatigue Severity Scale (FSS) among persons with multiple sclerosis. Archives of Rehabilitation, 13(4), 84-91.

2. Barak, S., Hutzler, Y., Dubnov-Raz, G., \& Achiron, A. (2016). Physical exercise for people with multiple sclerosis: effects, recommendations and barriers. Harefuah, 155(6), 364-9.

3. Bikmoradi, A., Zafari, A., Oshvandi, K., Mazdeh, M., \& Roshanaei, G. (2014). Effect of Progressive Muscle Relaxation on Severity of Pain in Patients with Multiple Sclerosis: a Randomized Controlled Trial. Hayat, 20(1), 2637.

4. Charvet, L. E., Shaw, M. T., Sherman, K., Haas, S., \& Krupp, L. B. (2018). Timed instrumental activities of daily living in multiple sclerosis: The test of everyday cognitive ability (TECA). Multiple Sclerosis and Related Disorders, 23, 69-73. https://doi.org/10.1016/j.msard.2018.05.001

5. Dayapoglu, N., \& Tan, M. (2012). Evaluation of the effect of progressive relaxation exercises on fatigue and sleep quality in patients with multiple sclerosis. The Journal of Alternative and Complementary Medicine, 18(10), 983-987. https://doi.org/10.1089/acm.2011.0390

6. Edwards, T., \& Pilutti, L. A. (2017). The effect of exercise training in adults with multiple sclerosis with severe mobility disability: a systematic review and future research directions. Multiple Sclerosis and Related Disorders, 16, 31-39. https://doi.org/10.1016/j.msard.2017.06.003

7. Ghaffari, S., Ahmadi, F., Nabavi, S. M., Me'marian, R., \& Kazem-Nejad, A. (2007). Effect of Applying Progressive Muscle Relaxation Activities of Daily Living in Multiple Sclerosis Patients. Journal of Rehabilitation, 8(2), 73-80.

8. Habibi Sola, A., Nikpoor, S., Rezaei, M., \& Haghani, H. (2007). Health Promotion Behaviours and Level of Activities of Daily Living and Instrumental Activities of Daily Living Among Elderly People in West Region of Tehran: A CrossSectional Survey. Iranian Journal of Ageing, 2(3), 331-339.

9. Hooshmand, A. (2018). The seventy thousand people in Iran have MS[Internet].Tehran:World Economy Newspaper; [updated 2018 june 19;cited 2018 december 6 Th]. Available from: https://www.donya-e-eqtesad.com/fa/tiny/news3402357.

10. Jalal manesh, S. O. M., \& Zargarani, F. (2015). Effects ofprogressive muscle relaxation technique on fatigue and sleep quality in patients with multiple sclerosis. Scientific Journal of Hamadan Nursing \& Midwifery Faculty, 23(3), 514.

11. Jariani, M., Saki, M., Momeni, N., Ebrahimzade, F., \& Seydian, A. (2011). The effect of progressive muscle relaxation techniques on anxiety in Patients with myocardial infarction. Yafteh 13(3), 22-30.

12. Kaminska, J., Koper, O. M., Piechal, K., \& Kemona, H. (2017). Multiple sclerosis - etiology and diagnostic potential. Postep Hig Med Dosw, 71, 551-563. https://doi.org/10.5604/01.3001.0010.3836

13. Kratz, A. L., Braley, T. J., Foxen-Craft, E., Scott, E., Murphy, J. F., \& Murphy, S. L. (2017). How Do Pain, Fatigue, Depressive, and Cognitive Symptoms Relate to Well-Being and Social and Physical Functioning in the Daily Lives of Individuals With Multiple Sclerosis? Archives of Physical Medicine and Rehabilitation, 98(11), 2160-2166. https://doi.org/10.1016/j.apmr.2017.07.004

14. Mikulakova, W., Klimova, E., Kendrova, L., Gajdos, M., \& Chmelik, M. (2018). Effect of Rehabilitation on Fatigue Level in Patients with Multiple Sclerosis. Medical Science Monitor, 24, 5761-70. https://doi.org/10.12659/MSM.909183

15. Rigi, F., shahnavazi, M., Feizi, A., Adineh, H., \& Malekraisi noushirvan, S. (2017). The Effect of Reflexology Massage On The Fatigue Severiy In Women With Multiple Sclerosise. Journal of Urmia Nursing and Midwifery Faculty, 14(11), 926-933.

16. Sebastião, E., Pilutti, L. A., Motl, R. W. (2019). Aerobic Fitness and Instrumental Activities of Daily Living in People with Multiple Sclerosis: A Cross-sectional Study. International Journal of MS Care, 21(1), 23. https://doi.org/10.7224/1537-2073.2017-078

17. Yilmaz, C. K., \& Kapucu, S. (2017). The Effect of Progressive Relaxation Exercises on Fatigue and Sleep Quality in Individuals with COPD. Holistic Nursing Practice, 31(6), https://doi.org/10.1097/HNP.0000000000000234

18. Zargarani, F., Maghsoudlou, A., \& Zaman Kamkar, M (2018). The effect of progressive muscle relaxation technique on the quality of sleep and fatigue in patients with multiple sclerosis. Journal of Urmia Nursing and Midwifery Faculty, 15(12), 911-920.

19. Zargarzadeh, M., Memarian, R., \& Rafiee, A. (2015). The effect of progressive muscle relaxation program on chronic low back pain and living daily activities in patients with intervertebral disc: a randomized clinical trial. Journal of Shahrekord University of Medical Sciences, 16(6), 101-10. 